\title{
Proteomic Profile of Circulating Immune Complexes in Dengue Infected Patients
} \author{
Kenji Hirayama ${ }^{1,9^{*}}$ \\ ${ }^{1}$ Department of Immunogenetics, Institute of Tropical Medicine (NEKKEN), Nagasaki University, Japan \\ ${ }^{2}$ Department of Clinical Product Development, Institute of Tropical Medicine (NEKKEN), Nagasaki University, Japan \\ ${ }^{3}$ Department of Virology, Institute of Tropical Medicine (NEKKEN), Nagasaki University, Japan \\ ${ }^{4}$ Laboratory of Arbovirus, Pasteur Institute in Ho Chi Minh City, Vietnam \\ ${ }^{5}$ Children's Hospital No.2, Ho Chi Minh City, Vietnam \\ ${ }^{6}$ Center for Preventive Medicine, Vinh Long, Vietnam \\ ${ }^{7}$ Course of Pharmaceutical Sciences, Graduate School of Biomedical Sciences, Nagasaki University, Nagasaki, Japan \\ ${ }^{8}$ Nagasaki University Research Centre for Genomic Instability and Carcinogenesis \\ (NRGIC), Nagasaki, Japan \\ ${ }^{9} \mathrm{Global}$ COE program, Nagasaki University, Japan \\ \#These authors contributed equally to this work
}

Nguyen Tien Huy ${ }^{1,2 \#}$, Huynh Trung Trieu ${ }^{1 \#,}$, Kenta Okamoto ${ }^{3}$, Tran Thi Hai Ninh ${ }^{1}$, Tran Thi Ngoc Ha ${ }^{1}$, Kouichi Morita ${ }^{3}$, Vu Thi Que Huong ${ }^{3}$ Nguyen Thi Phuong Lan ${ }^{4}$, Tran Thi Thuy ${ }^{5}$, Cao Thi Phi Nga ${ }^{6}$, Mihoko Kikuchi ${ }^{1}$, Naotaka Kuroda ${ }^{7}$, Juntra Karbwang ${ }^{2}$, Kaname Ohyama ${ }^{7} 8^{*}$, and

\begin{abstract}
Dengue virus is a flavivirus that causes Dengue Fever (DF), Dengue Hemorrhagic Fever (DHF), and Dengue Shock Syndrome (DSS), a serious public health problem in many countries. An auto-immune response is thought to play an important role in the pathogenesis of severe dengue and the increased level of Circulating Immune Complexes $(\mathrm{CIC})$ in dengue infected patients. Therefore, a proteomic analysis of proteins in the CIC can provide a better knowledge of the pathogenesis and a potential biomarker for severe dengue. A proteomic strategy based immune complexome analysis was performed to analyze the composition of $\mathrm{CIC}$ from plasma of fifteen dengue infected patients and five healthy control children. A total of 111 proteins were identified in the CIC from all individuals, with 17 proteins shared by healthy, DF, DHF, and DSS groups. All detected proteins were of similar relative proportion in the CIC of healthy, DF, DHF, and DSS groups. The results also revealed a high similarity of CIC profiles between four groups of subjects when classifying identified proteins according to cellular components or functional protein categories. These results showed no evidence to support the roles of $\mathrm{CIC}$ mediated by auto-immune response in the pathogenesis of severe dengue.
\end{abstract}

Keywords: Auto-immune; Circulating immune complexes; Dengue; DSS; Proteome; Severity

\section{Introduction}

Dengue infection has been becoming a serious public health problem in many countries with a dramatic increase globally. There are approximately 2.5 billion people at risk of dengue in over 100 countries. It is estimated that over 20,000 deaths occur every year due to this disease [1]. Dengue hemorrhagic fever (DHF) is the severe form of dengue infection, which is characterized by plasma leakage possibly inducing hypovolemic shock, known as dengue shock syndrome (DSS). Some patients infected by dengue virus develop dengue fever (DF), some develop DHF and about $20-30 \%$ of them, who suffer from DHF, develop shock [2]. At present, there is no approved dengue vaccine nor antiviral drug, although some potential solutions are currently being studied [3]. Early treatment, vector control, and educational program are the only methods to reduce global disease burden and mortality [47]. Therefore, it is important to understand the pathogenesis of dengue infection in order to find an appropriate management.

There are many factors contributing the pathogenesis of dengue virus infection, including virulence factor, secondary infection [8], host genetic factors [9-11], host immune response [12-14] and physiological factors [15]. An auto-immune response has also been proposed as an underlying mechanism in the pathogenesis of dengue infection [16-22]. In this hypothesis, immune complexes (IC) formed by auto-antibodies and human proteins are the main feature resulting in severity of disease. Ohyama et al. proposed a novel proteomic strategy (immune complexome analysis) that entails the separation of CICs from blood, direct tryptic digestion, and nano-liquid chromatography-tandem mass spectrometry [23]. They analyzed the CICs in rheumatoid arthritis which is a representative autoimmune disease and found two CICs which includes antigens specifically detected in that disease [23,24]. Therefore, it is important to analyze the composition of CIC in dengue infected patients not only to be used as diagnostic tools, but also to understand new molecular pathways involved in diseases. In this study, an immune complexome analysis of plasma from different groups of healthy, DF, DHF, and DSS were performed and compared using a proteomic approach.

\section{Materials and Methods}

\section{Study design}

The current study was performed at the Infectious Department

*Corresponding authors: Kaname Ohyama, Course of Pharmaceutical Sciences Nagasaki University Research Centre for Genomic Instability and Carcinogenesis, Nagasaki University, Japan, E-mail: kohyama@nagasaki-u.ac.jp

Kenji Hirayama, Department of Immunogenetics, Institute of Tropical Medicine (NEKKEN), Nagasaki University, Japan, E-mail: hiraken@nagasaki-u.ac.jp

Received June 13, 2013; Accepted June 24, 2013; Published June 27, 2013

Citation: Huy NT, Trieu HT, Okamoto K, Ninh TTH, Ha TTN, et al. (2013) Proteomic Profile of Circulating Immune Complexes in Dengue Infected Patients. J Trop Dis 1: 109. doi:10.4172/2329-891X.1000109

Copyright: (C) 2013 Huy NT, et al. This is an open-access article distributed unde the terms of the Creative Commons Attribution License, which permits unrestricted use, distribution, and reproduction in any medium, provided the original author and source are credited. 
of Pediatric Hospital Number 2, Ho Chi Minh City, and the Center for Preventive Medicine in Vinh Long province, Vietnam. It was a hospital-based case control study in children aged 6 months to 15 years with suspected dengue infections from 2006 to 2007. The study was approved by the institutional ethical review committees of the Institute of Tropical Medicine, Nagasaki University, Pediatric Hospital Number 2, Center for Preventive Medicine in Vinh Long, and the Pasteur Institute in Ho Chi Minh City. Written informed consent was required from the parents or guardians on the behalf of all children participants involved in the study. All experiments were conducted in accordance with the Declaration of Helsinki.

The entry criteria were children with suspected dengue infection based on clinical symptoms. After admission, the patients were diagnosed using standardized dengue virus isolation, serology techniques, and RT-PCR assay as previously described [10,25]. A positive confirmed laboratory test was made when the result of dengue virus isolation was positive or RT-PCR assay determined a dengue serotype, or when there was a positive anti-DV IgM antibody-capture ELISA, a positive seroconversion, or a $\geq 4$-fold increase in anti-DV IgG titres between acute and convalescent samples. The molecular detection of the dengue virus genome was performed using a ReadyTo-Go reverse transcriptase PCR test kit (Amersham, MA, USA) [26]. Dengue virus isolation was carried out on the C6/36 cell line and viral identification was detected by a direct and indirect fluorescent antibody technique with monoclonal antibodies supplied by the Centers for Disease Control and Prevention (For Collins, CO, USA) [27]. Serological assays for anti-DV IgM and IgG by IgM-and IgG-capture ELISA were conducted by an in-house Kit of the Pasteur Institute (HCMC) on both the acute and convalescent plasma samples, collected

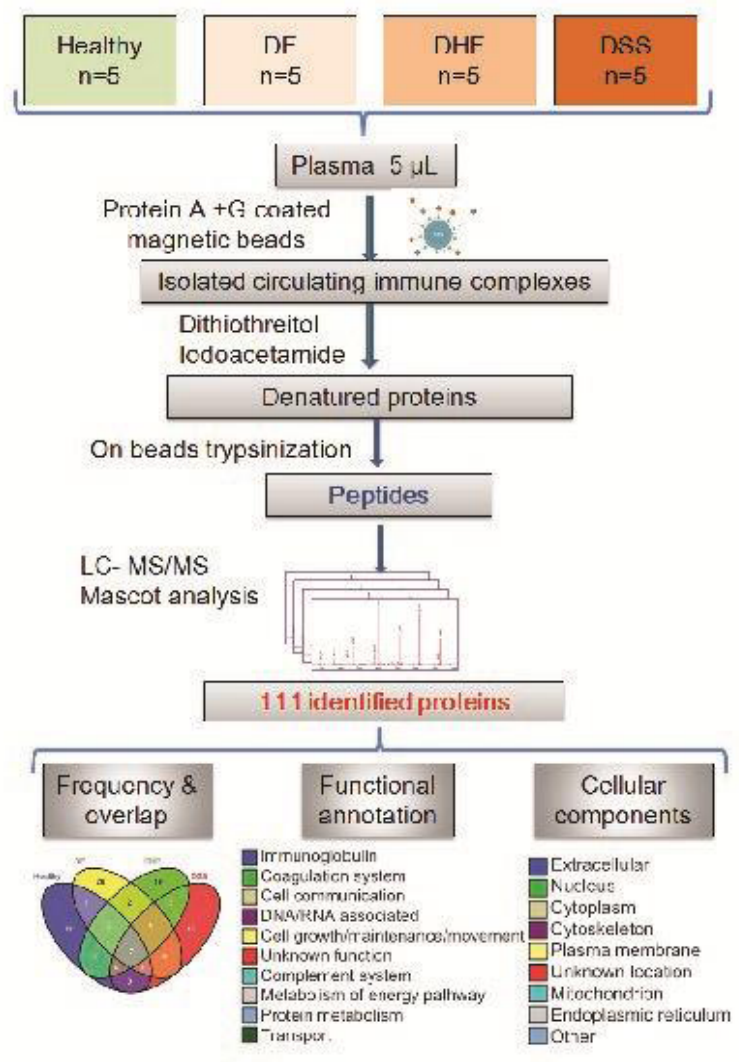

Figure 1: Flow diagram of methodology in this study. at $\geq 3$-day intervals [28]. The cases were defined as secondary infection if the DV IgM/IgG ratio was $<1.8$.

The severity of the disease was classified according to the WHO (1997) classification criteria for dengue virus infection [29]. Plasma samples were obtained from five patients in each groups of DF, DHF, and DSS patients during the transition period of fever to defervescence. In addition, school children living in Ho Chi Minh City who had no symptoms of any diseases and a negative standardized dengue serological test were chosen as a healthy control group. Five samples in each group have been suggested as the minimal number of samples in the shotgun proteomic study [30]. This number has been also used in several proteomic analysis [31-33].

\section{Sample collection and preparation}

Blood samples were drawn into EDTA tubes. Plasma was separated by centrifugation at $3000 \mathrm{rpm}$ for $10 \mathrm{~min}$, stored at $-80^{\circ} \mathrm{C}$ and centrifuged again at $3000 \mathrm{rpm}$ for $10 \mathrm{~min}$ before being used for CIC isolation.

CIC was isolated by magnetic beads with immobilized protein $\mathrm{A} / \mathrm{G}$ (an equal mixture of PureProteome ${ }^{\mathrm{ms}}$ Protein A and PureProteome ${ }^{\mathrm{mm}}$ Protein G Magnetic Bead Systems; Millipore) as previously described [23] and illustrated in Figure 1. Briefly, plasma $(5 \mu \mathrm{L})$ was diluted with $90 \mu \mathrm{L}$ PBS $\left(9.0 \mathrm{mM} \mathrm{Na}_{2} \mathrm{HPO}_{4}, 2.9 \mathrm{mM} \mathrm{NaH} 2 \mathrm{PO} 4\right.$, and $137 \mathrm{mM}$ $\mathrm{NaCl})$ and incubated with magnetic beads $(20 \mu \mathrm{L})$ for $30 \mathrm{~min}$ at room temperature with gentle mixing. The unbound fraction was washed 3 times with $500 \mu \mathrm{L}$ PBS using a magnet. The beads with bound CIC were recovered and resuspended in $100 \mu \mathrm{L}$ of $10 \mathrm{mM}$ dithiothreitol and incubated at $56^{\circ} \mathrm{C}$ for $45 \mathrm{~min}$. The sample was next added by $100 \mu \mathrm{L}$ of $55 \mathrm{mM}$ iodoacetamide and incubated at room temperature for $30 \mathrm{~min}$ in the dark. Trypsin (Promega) was further added into the sample at a final concentration of $0.5 \mathrm{mg} / \mathrm{mL}$. After an overnight incubation at $37^{\circ} \mathrm{C}$, the sample was subsequently added with $5 \mu \mathrm{L}$ of $5 \%$ formic acid to stop the digestion. The supernatant containing the peptide digests of CIC was dried by a centrifugal vacuum evaporator. The sample was dissolved in $10 \mu \mathrm{L}$ of $0.3 \%$ formic acid and was centrifuged at $20,000 \times \mathrm{g}$ for $10 \mathrm{~min}$ to collect $5 \mu \mathrm{L}$ of supernatant for injection into the LC-MS/ MS analysis.

\section{Mass Spectrometric Analysis and Database Search}

The MS and tandem-MS (MS/MS) spectra of trypsinized peptides were obtained using the NanoFrontier $\mathrm{nLC}$ and NanoFrontier eLD Liquid Chromatography Mass Spectrometer (Hitachi Hightechnologies, Tokyo, Japan). The nano-Liquid Chromatography/ ElectroSpray Ionization/ Linear Ion Trap/ Time of Flight (nLC-ESI/LIT/ TOF) and collision induced dissociation (CID) modes were used for MS detection and peptide fragmentation as previously described [34] In the nLC-ESI/LIT/TOF, the trypsinized peptides $(5 \mu \mathrm{L})$ were trapped on monolith trap column [C18-50-150 column, (0.05 mm I.D. $\times 150$ $\mathrm{mm} \mathrm{L}$ ). Hitachi High-technologies] and separated by a nano-capillary column [NTCC-360/75-3-123, (0.075 mm I.D. $\times 100 \mathrm{~mm} \mathrm{~L}$, particle diameter $3 \mu \mathrm{m}$ ), Nikkyo Technos Co., Ltd, Tokyo, Japan] at a flow rate of $200 \mathrm{~nL} / \mathrm{min}$. The peptides were then eluted using a stepwise acetonitrile (ACN) gradient (mobile phase A: $2 \% \mathrm{ACN}, 0.1 \%$ formic acid; mobile phase B: $98 \% \mathrm{ACN}, 0.1 \%$ formic acid, the A: B concentration gradient was 100:0 at zero min and 0:100 at $60 \mathrm{~min}$, respectively). In the nLCESI/LIT/TOF system, the eluted peptides were ionized with a capillary voltage of $1700 \mathrm{~V}$ and detected in a detector potential TOF range of 2050-2150 V.

Raw MS and MS/MS spectra were converted into Mascot generic 
format (mgf) using a Data Processing software 2008 (Hitachi Hightechnologies) and subsequently searched using the MS/MS Ion Search provided by MASCOT Sequence Query sever version 2.3 against the Swiss-Prot database (human and dengue virus only). The following search parameters were used, enzyme: trypsin, variable modifications: carbamidomethylation (C) and oxidation (M), mass values: monoisotopic, protein mass: unrestricted, peptide mass tolerance: \pm 0.5 $\mathrm{Da}$, fragment mass tolerance: $\pm 0.2 \mathrm{Da}$ (CID data), maximum missed cleavages: 1 and Instrument type: ESI-TRAP.

For MASCOT output, significant peptides were determined by the peptides score from the probability-based molecular weight search (MOWSE) which identifies proteins from the molecular weight of peptides created by the trypsin digestion [35]. Peptide score $>25$ indicated an identity or extensive homology $(p<0.05)$. Further stringency was added by eliminating any single peptide that could be assigned to more than one protein. The protein identifications were further checked manually in the database for possible redundancies including multiple names and homologies. Keratins and trypsin were considered as contaminating proteins and were excluded from our analysis. The Venn diagrams were created using a web-based Venny program [36] (Figure 1).

\section{Functional annotation of identified proteins}

Identified proteins of all individuals in each group were combined and were characterized into molecular functions and cellular components using an online based UniProt-GOA program.

\section{Statistical Analysis}

Kruskal-Wallis test was used for comparison of three or more unmatched groups. Fisher's exact test was used for pairwise comparison of two unmatched groups as the sample size was small in each group. The difference was considered significant at $\mathrm{p}<0.05$.

\section{Results}

The schematized Fig. 1 gives information on the design and experimental procedures. A total of 20 subjects, including $5 \mathrm{DF}, 5 \mathrm{DHF}$, 5 DSS patients, and 5 healthy children were enrolled in this study, and their characteristics are summarized in Table 1. All plasma samples of

\begin{tabular}{|c|c|c|c|c|}
\hline & HT & DF & DHF & DSS \\
\hline Number of patients & 5 & 5 & 5 & 5 \\
\hline $\mathrm{Age}^{a}$ & $5(3-8)$ & $10(5-13)$ & $9(8-11)$ & $7(5-11)$ \\
\hline Male : Female & $3: 2$ & $1: 4$ & $4: 1$ & $1: 4$ \\
\hline Day of illness on admission ${ }^{a}$ & & $4(2-4)$ & $4(4-4)$ & $4(3-5)$ \\
\hline Day of sampling ${ }^{a}$ & & $4(3-5)$ & $4(4-4)$ & $4(3-5)$ \\
\hline \multicolumn{5}{|l|}{ Serology diagnosis } \\
\hline Primary infection & & 0 & 2 & 0 \\
\hline Secondary infection & & 5 & 3 & 5 \\
\hline \multicolumn{5}{|l|}{ Dengue serotype } \\
\hline DEN-1 & & 1 & 2 & 3 \\
\hline DEN-2 & & 1 & 1 & 1 \\
\hline DEN-3 & & & & 1 \\
\hline
\end{tabular}

a Median (minimum, maximum),

$H T$, healthy children; DF, dengue fever; DHF, dengue hemorrhagic fever; DSS, dengue shock syndrome

Table 1: Clinical characteristics of subjects.

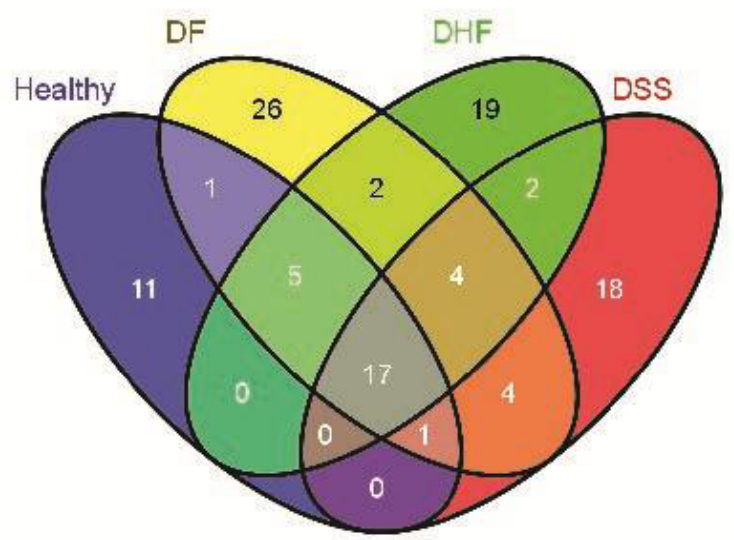

Figure 2: Overlap of identified proteins in different groups. Venn diagram showing total proteins identified in at least one individual from each group of healthy (blue), DF (yellow), DHF (green), and DSS (red). Numbers of identified proteins were shown in different overlaps between groups. Table 2 shows the lists of identified proteins in this study.

dengue patients were collected during the transition period of fever to defervescence (day 3-5), which were not significantly different between dengue groups ( $\mathrm{p}$-value $>0.10$, Kruskal-Wallis test).

An immune complexome analysis of plasma from patients with dengue virus infection and healthy individuals were performed. A total of 35, 60, 49, and 46 proteins were identified in the healthy, DF, DHF, and DSS groups, respectively, resulting in a total identification of 111 identified proteins (Figure 2 and Table 2). Analysis of the Venn diagrams showed that only 17 identified proteins were overlapped in all groups of DF, DHF, and DSS (Figure 2). Among identified proteins $(n=18)$ that were detected in only patients with DSS, only one protein (YLP motif-containing protein 1) appeared in two patients, while other 17 proteins appeared in only one patient with DSS. Four proteins (serum albumin, complement C4-A, immunoglobulin J chain, and nesprin-1) were detected in patients with DF, DHF, and DSS but not in healthy individuals, however, all of four proteins were only identified in less than three of five patients in each group of DF, DHF, and DSS. All detected proteins were of similar relative frequency in the circulating immune complexome of healthy, DF, DHF, and DSS groups ( $p$-value $>0.10$, Kruskal-Wallis test). Pairwise comparison of all detected proteins showed no significant difference in the frequency of particular protein between groups ( $\mathrm{p}$-value $>0.10$, Fisher's exact test).

Two proteins including Rho GTPase-activating protein 18 and Ubiquitin-conjugating enzyme E2 variant 3 were detected in one DHF and DSS patients but not in any DF/health individual. In addition, no significant differences in the relative frequency of detected proteins were found between the severe dengue groups (DHF/DSS) and the DF/ healthy groups ( $\mathrm{p}$-value $>0.10$, Fisher's exact test).

Functional analysis by UniProt-GOA program revealed ten protein classes including immunoglobulin, coagulation system, cell communication, DNA/RNA association, cell growth/maintenance/ movement, complement, energy metabolism, protein metabolism, and transport system (Figure 3). All ten functional classes were found in all groups of the healthy, DF, DHF, and DSS. The immunoglobulin class accounted the highest number of proteins in all groups, followed by coagulation system, cell communication, and DNA/RNA associated classes. There are no significant differences in the percentage of numbers of proteins found per group in any functional protein class (p-value $>0.10$, Kruskal-Wallis test). 
Citation: Huy NT, Trieu HT, Okamoto K, Ninh TTH, Ha TTN, et al. (2013) Proteomic Profile of Circulating Immune Complexes in Dengue Infected Patients. J Trop Dis 1: 109. doi:10.4172/2329-891X.1000109

Page 4 of 8

\begin{tabular}{|c|c|c|c|c|c|c|c|}
\hline \multirow{2}{*}{ No. } & \multirow{2}{*}{ Protein ID } & \multirow{2}{*}{ Protein } & \multirow{2}{*}{ MW } & \multicolumn{4}{|c|}{ Frequency } \\
\hline & & & & Healthy & DF & DHF & DSS \\
\hline 1 & ALBU_HUMAN & Serum albumin ${ }^{c}$ & 69321 & $0 / 5$ & $1 / 5$ & $1 / 5$ & $2 / 5$ \\
\hline 2 & ARID2_HUMAN & AT-rich interactive domain-containing protein $2^{b}$ & 197268 & $0 / 5$ & $0 / 5$ & $0 / 5$ & $1 / 5$ \\
\hline 3 & ASXL1_HUMAN & Additional sex combs like 1 & 165432 & $0 / 5$ & $1 / 5$ & $0 / 5$ & $0 / 5$ \\
\hline 4 & AT7L3_HUMAN & Ataxin-7-like protein 3 & 38651 & $0 / 5$ & $1 / 5$ & $0 / 5$ & $1 / 5$ \\
\hline 5 & BPAEA_HUMAN & Bullous pemphigoid antigen 1 , isoforms $6 / 9 / 10$ & 590626 & $0 / 5$ & $1 / 5$ & $0 / 5$ & $0 / 5$ \\
\hline 6 & BPTF_HUMAN & Nucleosome-remodeling factor subunit BPTF & 338262 & $0 / 5$ & $1 / 5$ & $1 / 5$ & $0 / 5$ \\
\hline 7 & BRD7_HUMAN & Bromodomain-containing protein 7 & 74092 & $0 / 5$ & $1 / 5$ & $1 / 5$ & $0 / 5$ \\
\hline 8 & C1QB_HUMAN & Complement $\mathrm{C} 1 \mathrm{q}$ subcomponent subunit $\mathrm{B}^{\mathrm{b}}$ & 26442 & $0 / 5$ & $0 / 5$ & $0 / 5$ & $1 / 5$ \\
\hline 9 & C1QC_HUMAN & Complement $\mathrm{C} 1 \mathrm{q}$ subcomponent subunit $\mathrm{C}^{\mathrm{a}}$ & 25757 & $3 / 5$ & $3 / 5$ & $3 / 5$ & $1 / 5$ \\
\hline 10 & C1QR1_HUMAN & Complement component $\mathrm{C} 1 \mathrm{q}$ receptor & 68515 & $0 / 5$ & $0 / 5$ & $1 / 5$ & $0 / 5$ \\
\hline 11 & CB016_HUMAN & Uncharacterized protein C2orf16 & 224321 & $0 / 5$ & $1 / 5$ & $0 / 5$ & $0 / 5$ \\
\hline 12 & CCD68_HUMAN & Coiled-coil domain-containing protein 68 & 38845 & $1 / 5$ & $3 / 5$ & $3 / 5$ & $0 / 5$ \\
\hline 13 & CE042_HUMAN & Uncharacterized protein C5orf42 & 236516 & $0 / 5$ & $1 / 5$ & $0 / 5$ & $0 / 5$ \\
\hline 14 & CHRD_HUMAN & Chordin & 101966 & $0 / 5$ & $1 / 5$ & $0 / 5$ & $1 / 5$ \\
\hline 15 & CK042_HUMAN & Uncharacterized protein C11orf42 & 36358 & $0 / 5$ & $1 / 5$ & $0 / 5$ & $0 / 5$ \\
\hline 16 & CO4A_HUMAN & Complement $\mathrm{C} 4-\mathrm{A}^{\mathrm{C}}$ & 192650 & $0 / 5$ & $1 / 5$ & $2 / 5$ & $2 / 5$ \\
\hline 17 & COBA1_HUMAN & Collagen alpha- $1(\mathrm{XI})$ chain & 180954 & $1 / 5$ & $2 / 5$ & $1 / 5$ & $1 / 5$ \\
\hline 18 & CP2E1_HUMAN & Cytochrome P450 2E1 & 56812 & $0 / 5$ & $1 / 5$ & $0 / 5$ & $0 / 5$ \\
\hline 19 & CSTFT_HUMAN & Cleavage stimulation factor $64 \mathrm{kDa}$ subunit, tau variant & 64396 & $0 / 5$ & $1 / 5$ & $0 / 5$ & $0 / 5$ \\
\hline 20 & DI3L1_HUMAN & DIS3-like exonuclease $1^{b}$ & 120711 & $0 / 5$ & $0 / 5$ & $0 / 5$ & $1 / 5$ \\
\hline 21 & DMD_HUMAN & Dystrophin & 426692 & $0 / 5$ & $1 / 5$ & $0 / 5$ & $0 / 5$ \\
\hline 22 & DOCK2_HUMAN & Dedicator of cytokinesis protein $2^{\mathrm{d}}$ & 211948 & $1 / 5$ & $0 / 5$ & $0 / 5$ & $0 / 5$ \\
\hline 23 & DYH6_HUMAN & Dynein heavy chain 6 , axonemal & 475982 & $0 / 5$ & $0 / 5$ & $1 / 5$ & $0 / 5$ \\
\hline 24 & DYHC1_HUMAN & Cytoplasmic dynein 1 heavy chain $1^{\mathrm{b}}$ & 532072 & $0 / 5$ & $0 / 5$ & $0 / 5$ & $1 / 5$ \\
\hline 25 & ELMO1_HUMAN & Engulfment and cell motility protein $1^{\mathrm{b}}$ & 83829 & $0 / 5$ & $0 / 5$ & $0 / 5$ & $1 / 5$ \\
\hline 26 & EMIL3_HUMAN & EMILIN-3 & 82596 & $0 / 5$ & $0 / 5$ & $1 / 5$ & $0 / 5$ \\
\hline 27 & EPHAA_HUMAN & Ephrin type-A receptor 10 & 109716 & $0 / 5$ & $1 / 5$ & $0 / 5$ & $0 / 5$ \\
\hline 28 & FA156_HUMAN & Protein FAM156A & 24411 & $0 / 5$ & $0 / 5$ & $1 / 5$ & $0 / 5$ \\
\hline 29 & FGD6_HUMAN & FYVE, RhoGEF and PH domain-containing protein 6 & 160816 & $0 / 5$ & $1 / 5$ & $0 / 5$ & $0 / 5$ \\
\hline 30 & FIBA_HUMAN & Fibrinogen alpha chain & 94914 & $5 / 5$ & $5 / 5$ & $5 / 5$ & $5 / 5$ \\
\hline 31 & FIBB_HUMAN & Fibrinogen beta chain & 55892 & $5 / 5$ & $5 / 5$ & $5 / 5$ & $5 / 5$ \\
\hline 32 & FIBG_HUMAN & Fibrinogen gamma chain & 51479 & $5 / 5$ & $5 / 5$ & $5 / 5$ & $4 / 5$ \\
\hline 33 & FUK_HUMAN & L-fucose kinase & 117623 & $1 / 5$ & $2 / 5$ & $1 / 5$ & $0 / 5$ \\
\hline 34 & GLI1_HUMAN & Zinc finger protein GLI1 ${ }^{\mathrm{b}}$ & 117904 & $0 / 5$ & $0 / 5$ & $0 / 5$ & $1 / 5$ \\
\hline 35 & HAIR_HUMAN & Protein hairless & 127495 & $0 / 5$ & $0 / 5$ & $1 / 5$ & $0 / 5$ \\
\hline 36 & HKR1_HUMAN & Krueppel-related zinc finger protein $1^{\mathrm{b}}$ & 75080 & $0 / 5$ & $0 / 5$ & $0 / 5$ & $1 / 5$ \\
\hline 37 & HRG_HUMAN & Histidine-rich glycoprotein & 59541 & $0 / 5$ & $0 / 5$ & $1 / 5$ & $0 / 5$ \\
\hline 38 & HV304_HUMAN & Ig heavy chain V-III region TIL & 12348 & $0 / 5$ & $1 / 5$ & $0 / 5$ & $0 / 5$ \\
\hline 39 & HV305_HUMAN & Ig heavy chain V-III region BRO & 13218 & $1 / 5$ & $1 / 5$ & 0/5 & $1 / 5$ \\
\hline 40 & IGHA1_HUMAN & Ig alpha-1 chain $C$ region & 37631 & $1 / 5$ & $2 / 5$ & $3 / 5$ & $3 / 5$ \\
\hline 41 & IGHG1_HUMAN & Ig gamma-1 chain $C$ region & 36083 & $5 / 5$ & $5 / 5$ & $5 / 5$ & $5 / 5$ \\
\hline 42 & IGHG2_HUMAN & Ig gamma-2 chain $C$ region & 35878 & $2 / 5$ & $2 / 5$ & $3 / 5$ & $1 / 5$ \\
\hline 43 & IGHG3_HUMAN & Ig gamma-3 chain $\mathrm{C}$ region & 41260 & $4 / 5$ & $5 / 5$ & $5 / 5$ & $4 / 5$ \\
\hline 44 & IGHG4_HUMAN & Ig gamma-4 chain $C$ region & 35918 & $2 / 5$ & $1 / 5$ & $1 / 5$ & $0 / 5$ \\
\hline 45 & IGHM_HUMAN & Ig mu chain $\mathrm{C}$ region & 49276 & $3 / 5$ & $5 / 5$ & $4 / 5$ & $3 / 5$ \\
\hline 46 & IGJ_HUMAN & Immunoglobulin J chain ${ }^{\mathrm{c}}$ & 15585 & $0 / 5$ & $1 / 5$ & $2 / 5$ & $1 / 5$ \\
\hline 47 & IGKC_HUMAN & Ig kappa chain $\mathrm{C}$ region & 11602 & $5 / 5$ & $5 / 5$ & $5 / 5$ & $5 / 5$ \\
\hline 48 & INSR_HUMAN & Insulin receptor & 156206 & $0 / 5$ & $3 / 5$ & $0 / 5$ & $2 / 5$ \\
\hline 49 & ITPR3_HUMAN & Inositol 1,4,5-trisphosphate receptor type $3^{\mathrm{b}}$ & 303912 & $0 / 5$ & $0 / 5$ & $0 / 5$ & $1 / 5$ \\
\hline 50 & JPH2_HUMAN & Junctophilin-2 $^{\mathrm{d}}$ & 74221 & $1 / 5$ & $0 / 5$ & $0 / 5$ & $0 / 5$ \\
\hline 51 & K0494_HUMAN & EF-hand domain-containing protein KIAA0494 b & 54997 & $0 / 5$ & $0 / 5$ & $0 / 5$ & $1 / 5$ \\
\hline 52 & K0753_HUMAN & Uncharacterized protein KIAA0753 ${ }^{\mathrm{b}}$ & 109350 & $0 / 5$ & $0 / 5$ & $0 / 5$ & $1 / 5$ \\
\hline 53 & KNG1_HUMAN & Kininogen-1 & 71957 & $1 / 5$ & $1 / 5$ & $0 / 5$ & $0 / 5$ \\
\hline 54 & KV106_HUMAN & Ig kappa chain V-I region EU & 11781 & $0 / 5$ & $1 / 5$ & $0 / 5$ & $0 / 5$ \\
\hline 55 & KV201_HUMAN & Ig kappa chain V-II region Cum & 12668 & $0 / 5$ & $2 / 5$ & $0 / 5$ & $0 / 5$ \\
\hline 56 & KV301_HUMAN & Ig kappa chain V-III region B6 & 11628 & $1 / 5$ & $1 / 5$ & $1 / 5$ & $0 / 5$ \\
\hline 57 & KV302_HUMAN & Ig kappa chain V-III region SIE & 11768 & $2 / 5$ & $2 / 5$ & $1 / 5$ & $2 / 5$ \\
\hline 58 & L2HDH_HUMAN & L-2-hydroxyglutarate dehydrogenase, mitochondrial ${ }^{b}$ & 50327 & $0 / 5$ & $0 / 5$ & $0 / 5$ & $1 / 5$ \\
\hline
\end{tabular}


Citation: Huy NT, Trieu HT, Okamoto K, Ninh TTH, Ha TTN, et al. (2013) Proteomic Profile of Circulating Immune Complexes in Dengue Infected Patients. J Trop Dis 1: 109. doi:10.4172/2329-891X.1000109

Page 5 of 8

\begin{tabular}{|c|c|c|c|c|c|c|c|}
\hline 59 & LAC_HUMAN & Ig lambda chain $\mathrm{C}$ regions & 11230 & $4 / 5$ & $5 / 5$ & $4 / 5$ & $5 / 5$ \\
\hline 60 & LRP1_HUMAN & Prolow-density lipoprotein receptor-related protein 1 & 504605 & $0 / 5$ & $0 / 5$ & $1 / 5$ & $0 / 5$ \\
\hline 61 & LTBP3_HUMAN & Latent-transforming growth factor beta-binding protein 3 & 139359 & $0 / 5$ & $1 / 5$ & $0 / 5$ & $0 / 5$ \\
\hline 62 & LV302_HUMAN & Ig lambda chain V-III region LOIb & 11928 & $0 / 5$ & $0 / 5$ & $0 / 5$ & $1 / 5$ \\
\hline 63 & LYG2_HUMAN & Lysozyme g-like protein 2 & 23498 & $0 / 5$ & $1 / 5$ & $0 / 5$ & $0 / 5$ \\
\hline 64 & M2OM_HUMAN & Mitochondrial 2-oxoglutarate/malate carrier protein ${ }^{d}$ & 34062 & $1 / 5$ & $0 / 5$ & $0 / 5$ & $0 / 5$ \\
\hline 65 & MCM9_HUMAN & DNA replication licensing factor $\mathrm{MCM}^{\mathrm{d}}$ & 43983 & $1 / 5$ & $0 / 5$ & $0 / 5$ & $0 / 5$ \\
\hline 66 & MD12L_HUMAN & $\begin{array}{l}\text { Mediator of RNA polymerase II transcription subunit 12-like } \\
\text { protein }^{d}\end{array}$ & 239967 & $1 / 5$ & $0 / 5$ & $0 / 5$ & $0 / 5$ \\
\hline 67 & MECR_HUMAN & Trans-2-enoyl-CoA reductase, mitochondrial ${ }^{d}$ & 40462 & $1 / 5$ & $0 / 5$ & $0 / 5$ & $0 / 5$ \\
\hline 68 & MY15B_HUMAN & Putative myosin-XVB ${ }^{b}$ & 167013 & $0 / 5$ & $0 / 5$ & $0 / 5$ & $1 / 5$ \\
\hline 69 & MYH7B_HUMAN & Myosin-7B & 221252 & $0 / 5$ & $1 / 5$ & $0 / 5$ & $0 / 5$ \\
\hline 70 & MYH9_HUMAN & Myosin-9 & 226392 & $0 / 5$ & $1 / 5$ & $0 / 5$ & $1 / 5$ \\
\hline 71 & MYO7A_HUMAN & Myosin-VIla & 254245 & $0 / 5$ & $2 / 5$ & $0 / 5$ & $0 / 5$ \\
\hline 72 & NIPS2_HUMAN & Protein NipSnap homolog 2 & 33721 & $3 / 5$ & $2 / 5$ & $3 / 5$ & $3 / 5$ \\
\hline 73 & NP1L3_HUMAN & Nucleosome assembly protein 1-like 3 & 57593 & $0 / 5$ & $0 / 5$ & $1 / 5$ & $0 / 5$ \\
\hline 74 & NSD2_HUMAN & Probable histone-lysine N-methyltransferase NSD2 & 152258 & $0 / 5$ & $0 / 5$ & $1 / 5$ & $0 / 5$ \\
\hline 75 & NU153_HUMAN & Nuclear pore complex protein Nup153 & 153938 & $0 / 5$ & $1 / 5$ & $0 / 5$ & $0 / 5$ \\
\hline 76 & ODPX_HUMAN & $\begin{array}{l}\text { Pyruvate dehydrogenase protein } \mathrm{X} \text { component, } \\
\text { mitochondrial }^{\mathrm{b}}\end{array}$ & 54089 & $0 / 5$ & $0 / 5$ & $0 / 5$ & $1 / 5$ \\
\hline 77 & OR2G3_HUMAN & Olfactory receptor $2 \mathrm{G} 3$ & 34506 & $0 / 5$ & $1 / 5$ & $0 / 5$ & $0 / 5$ \\
\hline 78 & PB1_HUMAN & Protein polybromo- $1^{\mathrm{b}}$ & 192947 & $0 / 5$ & $0 / 5$ & $0 / 5$ & $1 / 5$ \\
\hline 79 & PCD23_HUMAN & Protocadherin-23 ${ }^{b}$ & 322034 & $0 / 5$ & $0 / 5$ & $0 / 5$ & $1 / 5$ \\
\hline 80 & PCNT_HUMAN & Pericentrin & 378037 & $0 / 5$ & $1 / 5$ & $0 / 5$ & $0 / 5$ \\
\hline 81 & PRKDC_HUMAN & DNA-dependent protein kinase catalytic subunit & 468788 & $2 / 5$ & $1 / 5$ & $3 / 5$ & $1 / 5$ \\
\hline 82 & PSD1_HUMAN & PH and SEC7 domain-containing protein 1 & 109475 & $0 / 5$ & $0 / 5$ & $1 / 5$ & $0 / 5$ \\
\hline 83 & RA51D_HUMAN & DNA repair protein RAD51 homolog 4 & 35027 & $0 / 5$ & $0 / 5$ & $1 / 5$ & $0 / 5$ \\
\hline 84 & RBM45_HUMAN & RNA-binding protein $45^{d}$ & 53346 & $1 / 5$ & $0 / 5$ & $0 / 5$ & $0 / 5$ \\
\hline 85 & RFPLB_HUMAN & Ret finger protein-like 4B & 29903 & $0 / 5$ & $0 / 5$ & $1 / 5$ & $0 / 5$ \\
\hline 86 & RHG18_HUMAN & Rho GTPase-activating protein 18 & 74900 & $0 / 5$ & $0 / 5$ & $1 / 5$ & $1 / 5$ \\
\hline 87 & RRBP1_HUMAN & Ribosome-binding protein 1 & 152381 & $0 / 5$ & $0 / 5$ & $1 / 5$ & $0 / 5$ \\
\hline 88 & RL36X_HUMAN & Putative 60 S ribosomal protein L36-like 1 & 12056 & $0 / 5$ & $0 / 5$ & $1 / 5$ & $0 / 5$ \\
\hline 89 & SPKAP_HUMAN & A-kinase anchor protein SPHKAP & 186339 & $1 / 5$ & $1 / 5$ & $1 / 5$ & $1 / 5$ \\
\hline 90 & SLK_HUMAN & STE20-like serine/threonine-protein kinase ${ }^{d}$ & 142695 & $1 / 5$ & $0 / 5$ & $0 / 5$ & $0 / 5$ \\
\hline 91 & SPAST_HUMAN & Spastin OS=Homo sapiens & 67155 & $0 / 5$ & $0 / 5$ & $1 / 5$ & $0 / 5$ \\
\hline 92 & ST18_HUMAN & Suppression of tumorigenicity 18 protein $^{\mathrm{b}}$ & 115083 & $0 / 5$ & $0 / 5$ & $0 / 5$ & $1 / 5$ \\
\hline 93 & STIM1_HUMAN & Stromal interaction molecule 1 & 77375 & $3 / 5$ & $3 / 5$ & $2 / 5$ & $3 / 5$ \\
\hline 94 & STRN4_HUMAN & Striatin-4 & 81266 & $0 / 5$ & $0 / 5$ & $1 / 5$ & $0 / 5$ \\
\hline 95 & SYAM_HUMAN & Probable alanyl-tRNA synthetase, mitochondrial & 107273 & $0 / 5$ & $0 / 5$ & $1 / 5$ & $0 / 5$ \\
\hline 96 & SYNE1_HUMAN & Nesprin-1 ${ }^{c}$ & 1010412 & $0 / 5$ & $1 / 5$ & $2 / 5$ & $1 / 5$ \\
\hline 97 & T22D1_HUMAN & TSC22 domain family protein 1 & 109592 & $0 / 5$ & $0 / 5$ & $0 / 5$ & $0 / 5$ \\
\hline 98 & TITIN_HUMAN & Titin & 3813810 & $0 / 5$ & $1 / 5$ & $0 / 5$ & $0 / 5$ \\
\hline 99 & UBN1_HUMAN & Ubinuclein & 121520 & $0 / 5$ & $1 / 5$ & $0 / 5$ & $0 / 5$ \\
\hline 100 & UBP33_HUMAN & Ubiquitin carboxyl-terminal hydrolase $33^{d}$ & 106727 & $1 / 5$ & $0 / 5$ & $0 / 5$ & $0 / 5$ \\
\hline 101 & UBP37_HUMAN & Ubiquitin carboxyl-terminal hydrolase $37^{\mathrm{d}}$ & 110144 & $1 / 5$ & $0 / 5$ & $0 / 5$ & $0 / 5$ \\
\hline 102 & UEVLD_HUMAN & Ubiquitin-conjugating enzyme E2 variant 3 & 52231 & $0 / 5$ & $0 / 5$ & $1 / 5$ & $1 / 5$ \\
\hline 103 & WNK4_HUMAN & Serine/threonine-protein kinase WNK4 & 134655 & $1 / 5$ & $2 / 5$ & $3 / 5$ & $0 / 5$ \\
\hline 104 & YLPM1_HUMAN & YLP motif-containing protein $1^{\mathrm{b}}$ & 219849 & $0 / 5$ & $0 / 5$ & $0 / 5$ & $2 / 5$ \\
\hline 105 & ZEP2_HUMAN & Transcription factor HIVEP2 d & 269052 & $1 / 5$ & $0 / 5$ & $0 / 5$ & $0 / 5$ \\
\hline 106 & ZN177_HUMAN & Zinc finger protein 177 & 36473 & $0 / 5$ & $1 / 5$ & $0 / 5$ & $0 / 5$ \\
\hline 107 & ZN226_HUMAN & Zinc finger protein 226 & 91921 & $0 / 5$ & $0 / 5$ & $1 / 5$ & $0 / 5$ \\
\hline 108 & ZN514_HUMAN & Zinc finger protein 514 & 45938 & $0 / 5$ & $1 / 5$ & $0 / 5$ & $0 / 5$ \\
\hline 109 & ZN561_HUMAN & Zinc finger protein 561 & 55161 & $0 / 5$ & $0 / 5$ & $1 / 5$ & $0 / 5$ \\
\hline 110 & ZN669_HUMAN & Zinc finger protein 669 & 52597 & $0 / 5$ & $1 / 5$ & $0 / 5$ & $0 / 5$ \\
\hline 111 & ZNF48_HUMAN & Zinc finger protein 48 & 67833 & $0 / 5$ & $1 / 5$ & $0 / 5$ & $0 / 5$ \\
\hline
\end{tabular}

DF, dengue fever; DHF, dengue hemorrhagic fever; DSS, dengue shock syndrome; MW, molecular weight.

${ }^{a}$ Identified proteins $(n=16)$ were detected in individuals of four groups of healthy, DF, DHF, and DSS

${ }^{b}$ Identified proteins $(n=18)$ were detected in only patients with DSS.

${ }^{c}$ Identified proteins $(n=4)$ were detected in patients with DF, DHF, and DSS but not in healthy individuals.

${ }^{\mathrm{d}}$ Identified proteins $(\mathrm{n}=11)$ were only detected in healthy individuals.

Table 2: Frequency of identified proteins in CIC isolated from plasma. 


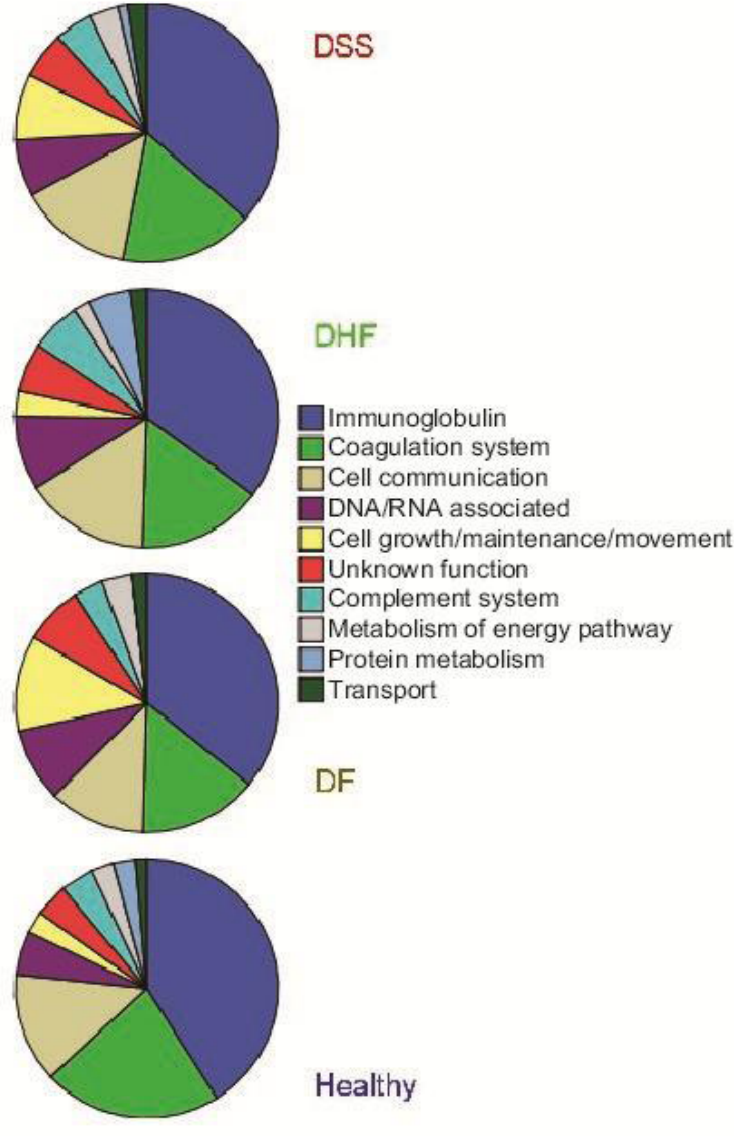

Figure 3: Functional analysis of identified proteins in different groups. Pie graph showing total proteins identified in at least one individual from each group and sorted by functional characteristics. The area in the graph represents the percentage of numbers of proteins found per group.

We further classified those identified proteins in terms of cellular components using the UniProt-GOA program. Figure 4 shows the proportions (\%) of proteins in different cellular components for each category of subjects. Three dominant protein classes are extracellular, nucleus, and cytoplasm. It is evidenced that other main cellular components including cytoskeleton, plasma membrane, mitochondrion, and endoplasmic reticulum also had identified proteins in the CIC. The results also indicated a high similarity of CIC profile between four groups when looking only at the cellular components. No significant differences were found when pairwise comparing the percentage of numbers of proteins in all cellular components' categories (p-value $>0.10$, Kruskal-Wallis test).

\section{Discussion}

Formation of CIC is a normal process of humoral immune response against an antigen. CIC is quickly uptake by monocytes, but in some situation they persist longer in the circulation or deposit in the local tissue, causing some pathology. It has been suggested that the IC can play an important role in pathogenesis of auto-immune diseases $[23,24,37]$. Moreover, CIC associated antigens have been detected as a hallmark of the auto-immune arthritis $[23,24]$.

An auto-immune response has been proposed as a mechanism in the pathogenesis of dengue infection, in which antibodies against dengue non-structural protein 1 (NS1) cross reacts with the host endothelial cells [16], platelets [17], active sites on human clotting factors and integrin/adhesin proteins [18]. Lin et al. [20] have detected that antibodies against NS1 cross-react with platelets and have higher binding activity to platelets in DHF/DSS than those in DF. Another study in Vietnamese children showed that levels of auto-antibodies against platelets and endothelial cells are higher in DHF/DSS compared to DF patients [21]. Level of CIC has been reportedly increased in dengue infection and peaked at the transition period of fever to defervescence [38]. The level of CIC is related to the severity of the disease. However, there were no strong evidences of (i) an association with other autoimmune diseases, (ii) infiltration of lymphocytes in the target site of the disease, and (iii) response to steroid treatment [39-41], which have been proposed as the criteria for an auto-immune pathogenesis [42].

In this study we found a similar relative composition of the CIC in

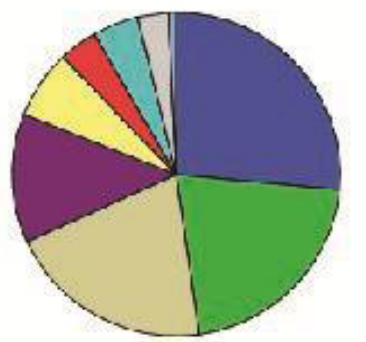

DSS
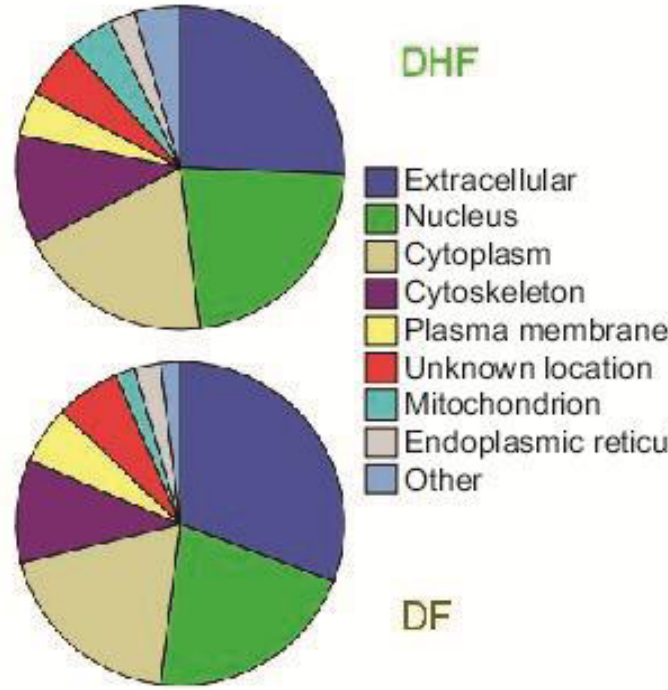

Unknown location

Mitochondrion

Endoplasmic reticulum

$\square$ Other

DF

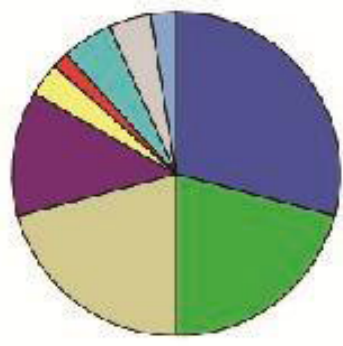

\section{Healthy}

Figure 4: Cellular component of identified proteins in different groups. Pie graph showing total proteins identified in at least one individual from each group and sorted by functional characteristics. Rare cellular components $(<3 \%)$ were grouped as 'other'. The area in the graph represents the percentage of numbers of proteins found per group. 
all groups of healthy, DF, DHF, and DSS which suggests the absence of any specific antigen consistently detectable during the transition from fever to defervescence. These results are in good agreement with the argument of Halstead [43], where he suggested that auto-antibodies would not play an important role in the pathogenesis of dengue severity because (i) the thrombocytopenia and hyper permeability occur in the early stage of the disease even in infant, while the antibody is produced later in the course of the disease [44]; (ii) the thrombocytopenia and hyperpermeability are transient while the production of antibody lasts for months [44]; (iii) the kinetics of antibody production in primary infections are completely different from secondary infections but the pathogenesis of DHF is not so much different between infants and children [8]. There was a limitation in this study such as the method could not detect non-protein substances of antigen including lipids and carbohydrates.

It is suggested that the lower sensitivity of dengue virus nonstructural protein-1 antigen (NS1) detection in secondary dengue infection compared with primary infection is due to the formation of CIC by anti-NS1 antibody IgG [45]. The dengue virus-containing immune complexes have been also detected using an immuno-precipitation assay coupled with a real-time RT-PCR method [46]. However, we didn't detect any dengue antigen including NS1 in the proteomic analysis of CIC, probably due to lower sensitivity of proteomic approach compare to the real-time RT-PCR method and a possible deposition of CIC at the local tissue. Thus, more sensitivity proteomic method is required for further studies to clarify this issue.

This study is the first to report a proteomic profile of circulating immune complexes from plasma of dengue infected patients. Our results showed similarity of CIC profiles between four groups of healthy, DF, DHF, and DSS when classifying identified proteins according to the frequency, cellular components or functional protein categories. Thus, it is unlikely that the CIC mediated by auto-immune response plays an important role in the pathogenesis of the acute dengue infection.

\section{Acknowledgments}

We thank all the members of the Laboratory of Arbovirus, Ho Chi Minh City, Vietnam. We also express our appreciation to the staff of Children's Hospital No. 2 in Ho Chi Minh City (HCMC) and the Center for Preventive Medicine in the Vinh Long Province (VL) of the Mekong Delta.

This work was supported in part by the Global COE Program (2008 - 2012) and Japan Initiative for Global Research Network on Infectious Diseases (J-GRID) for $\mathrm{KH}$. It was also supported in part by a "Grant-in-Aid for Scientific Research" from Nagasaki University to NTH (2012-2013).

\section{References}

1. WHO (2009) Epidemiology, burden of disease and trasmission. Dengue guidelines for diagnosis, treatment, prevention and control. 3-17.

2. WHO (2010) Management of Dengue Epidemic.

3. Gubler DJ (2011) Dengue, Urbanization and Globalization: The Unholy Trinity of the 21st Century. Trop Med Health 39: 3-11.

4. WHO (2009) World Health Organization. Guidelines for diagnosis, treatment, prevention and control; Organization WH, editor. Geneva: World Health Organization.

5. Wilder-Smith A, Ooi EE, Vasudevan SG, Gubler DJ (2010) Update on dengue: epidemiology, virus evolution, antiviral drugs, and vaccine development. Curr Infect Dis Rep 12: 157-164.

6. Kalayanarooj S (2011) Clinical Manifestations and Management of Dengue/ DHF/DSS. Trop Med Health 39: 83-87.

7. Al-Muhandis N, Hunter PR (2011) The value of educational messages embedded in a community-based approach to combat dengue Fever: a systematic review and meta regression analysis. PLoS Negl Trop Dis 5: e1278.
8. Halstead SB (2007) Dengue. Lancet 370: 1644-1652.

9. Lan NT, Hirayama K (2011) Host genetic susceptibility to severe dengue infection. Trop Med Health 39: 73-81.

10. Nguyen TP, Kikuchi M, Vu TQ, Do QH, Tran TT, et al. (2008) Protective and enhancing HLA alleles, HLA-DRB1*0901 and HLA-A*24, for severe forms of dengue virus infection, dengue hemorrhagic fever and dengue shock syndrome. PLoS Negl Trop Dis 2: e304.

11. Coffey LL, Mertens E, Brehin AC, Fernandez-Garcia MD, Amara A, et al. (2009) Human genetic determinants of dengue virus susceptibility. Microbes Infect 11 : 143-156.

12. Mongkolsapaya J, Duangchinda T, Dejnirattisai W, Vasanawathana $S$ Avirutnan P, et al. (2006) T cell responses in dengue hemorrhagic fever: are cross-reactive T cells suboptimal? J Immunol 176: 3821-3829.

13. Chaturvedi UC, Nagar R, Shrivastava R (2006) Macrophage and dengue virus: friend or foe? Indian J Med Res 124: 23-40.

14. Chaturvedi UC, Agarwal R, Elbishbishi EA, Mustafa AS (2000) Cytokine cascade in dengue hemorrhagic fever: implications for pathogenesis. FEMS Immunol Med Microbiol 28: 183-188.

15. Whitehorn J, Simmons CP (2011) The pathogenesis of dengue. Vaccine 29: 7221-7228.

16. Lin CF, Lei HY, Shiau AL, Liu CC, Liu HS, et al. (2003) Antibodies from dengue patient sera cross-react with endothelial cells and induce damage. J Med Virol 69: 82-90.

17. Huang KJ, Li SY, Chen SC, Liu HS, Lin YS, et al. (2000) Manifestation of thrombocytopenia in dengue-2-virus-infected mice. J Gen Virol 81: 2177-2182.

18. Falconar AK (1997) The dengue virus nonstructural-1 protein (NS1) generates antibodies to common epitopes on human blood clotting, integrin/adhesin proteins and binds to human endothelial cells: potential implications in haemorrhagic fever pathogenesis. Arch Virol 142: 897-916.

19. Lin CF, Wan SW, Cheng HJ, Lei HY, Lin YS (2006) Autoimmune pathogenesis in dengue virus infection. Viral Immunol 19: 127-132.

20. Lin CF, Lei HY, Liu CC, Liu HS, Yeh TM, et al. (2001) Generation of IgM antiplatelet autoantibody in dengue patients. J Med Virol 63: 143-149.

21. Hung NT, Lan NT, Lin Y-s, Lin C-f, Lien LB, et al. (2008) Anti-Platelet and Anti-Endothelial Cell Autoantibodies in Vietnamese Infants and Children with Dengue Hemorrhagic Fever American Journal of Infectious Diseases 4: 41-49.

22. Wan SW, Lin CF, Yeh TM, Liu CC, Liu HS, et al. (2013) Autoimmunity in dengue pathogenesis. J Formos Med Assoc 112: 3-11.

23. Ohyama K, Ueki Y, Kawakami A, Kishikawa N, Tamai M, et al. (2011) Immune complexome analysis of serum and its application in screening for immune complex antigens in rheumatoid arthritis. Clin Chem 57: 905-909.

24. Ohyama K, Kawakami A, Tamai M, Baba M, Kishikawa N, et al. (2012) Serum immune complex containing thrombospondin-1: a novel biomarker for early rheumatoid arthritis. Ann Rheum Dis 71: 1916-1917.

25. Ha TT, Huy NT, Murao LA, Lan NT, Thuy TT, et al. (2011) Elevated levels of cell-free circulating DNA in patients with acute dengue virus infection. PLoS One 6: e25969.

26. Lanciotti RS, Calisher CH, Gubler DJ, Chang GJ, Vorndam AV (1992) Rapid detection and typing of dengue viruses from clinical samples by using reverse transcriptase-polymerase chain reaction. J Clin Microbiol 30: 545-551.

27. Gubler DJ, Kuno G, Sather GE, Velez M, Oliver A (1984) Mosquito cell cultures and specific monoclonal antibodies in surveillance for dengue viruses. Am J Trop Med Hyg 33: 158-165.

28. Innis BL, Nisalak A, Nimmannitya S, Kusalerdchariya S, Chongswasdi V, et al. (1989) An enzyme-linked immunosorbent assay to characterize dengue infections where dengue and Japanese encephalitis co-circulate. Am J Trop Med Hyg 40: 418-427.

29. World Health Organization (1997) Dengue haemorrhagic fever: Diagnosis treatment, prevention and control. Geneva: World Health Organization.

30. Cairns DA, Barrett JH, Billingham LJ, Stanley AJ, Xinarianos G, et al. (2009) Sample size determination in clinical proteomic profiling experiments using mass spectrometry for class comparison. Proteomics 9: 74-86.

31. Lu YT, Han CL, Wu CL, Yu TM, Chien CW, et al. (2008) Proteomic profiles 
Citation: Huy NT, Trieu HT, Okamoto K, Ninh TTH, Ha TTN, et al. (2013) Proteomic Profile of Circulating Immune Complexes in Dengue Infected Patients. J Trop Dis 1: 109. doi:10.4172/2329-891X.1000109

Page 8 of 8

of bronchoalveolar lavage fluid from patients with ventilator-associated pneumonia by gel-assisted digestion and 2-D-LC/MS/MS. Proteomics Clin Appl 2: $1208-1222$.

32. Poon HF, Vaishnav RA, Getchell TV, Getchell ML, Butterfield DA (2006) Quantitative proteomics analysis of differential protein expression and oxidative modification of specific proteins in the brains of old mice. Neurobiol Aging 27: 1010-1019.

33. Bottoni P, Giardina B, Vitali A, Boninsegna A, Scatena R (2009) A proteomic approach to characterizing ciglitazone-induced cancer cell differentiation in Hep-G2 cell line. Biochim Biophys Acta 1794: 615-626.

34. Okamoto K, Endo Y, Inoue S, Nabeshima T, Nga PT, et al. (2010) Development of a rapid and comprehensive proteomics-based arboviruses detection system. J Virol Methods 167: 31-36.

35. Perkins DN, Pappin DJ, Creasy DM, Cottrell JS (1999) Probability-based protein identification by searching sequence databases using mass spectrometry data. Electrophoresis 20: 3551-3567.

36. Oliveros JC (2007) VENNY. An interactive tool for comparing lists with Venn Diagrams. BioinfoGP, CNB-CSIC.

37. Clough JD (1992) Role of autoantibodies and immune complexes in the pathogenesis of systemic lupus erythematosus. J Clin Apher 7: 151-152.

38. Ruangjirachuporn W, Boonpucknavig S, Nimmanitya S (1979) Circulating immune complexes in serum from patients with dengue haemorrhagic fever. Clin Exp Immunol 36: 46-53.
39. Tam DT, Ngoc TV, Tien NT, Kieu NT, Thuy TT, et al. (2012) Effects of shortcourse oral corticosteroid therapy in early dengue infection in Vietnamese patients: a randomized, placebo-controlled trial. Clin Infect Dis 55: 1216-1224.

40. Kularatne SA, Walathara C, Mahindawansa SI, Wijesinghe S, Pathirage MM, et al. (2009) Efficacy of low dose dexamethasone in severe thrombocytopenia caused by dengue fever: a placebo controlled study. Postgrad Med J 85: 525 529.

41. Panpanich R, Sornchai $P$, Kanjanaratanakorn K (2006) Corticosteroids fo treating dengue shock syndrome. Cochrane Database Syst Rev : CD003488.

42. Rose NR, Bona C (1993) Defining criteria for autoimmune diseases (Witebsky's postulates revisited) Immunol Today 14: 426-430.

43. Halstead SB (2008) Con: Dengue hemorrhagic fever is caused by autoimmune phenomena triggered by a dengue viral infection. In: Halstead SB, ed.: Dengue. London: Imperial College Press.

44. Halstead SB (2012) Controversies in dengue pathogenesis. Paediatr Int Child Health 32 Suppl 1: 5-9.

45. Tricou V, Minh NN, Farrar J, Tran HT, Simmons CP (2011) Kinetics of viremia and NS1 antigenemia are shaped by immune status and virus serotype in adults with dengue. PLoS Negl Trop Dis 5: e1309.

46. Wang WK, Chen HL, Yang CF, Hsieh SC, Juan CC, et al. (2006) Slower rates of clearance of viral load and virus-containing immune complexes in patients with dengue hemorrhagic fever. Clin Infect Dis 43: 1023-1030. 